\title{
Rett and ICF syndromes: methylation moves into medicine
}

Two human genetic disorders, Rett and ICF syndromes, have recently been shown to be caused by mutations in genes encoding proteins involved in gene silencing through DNA methylation. Rett (RTT) syndrome is a progressive childhood neurodevelopmental disorder that affects females exclusively with an incidence of 1 in 10,000-15,000 female births. Patients with RTT syndrome appear to develop normally until 6-18 months of age, then gradually loose speech and purposeful hand use and display a whole range of symptoms. These include microcephaly, seizures, autism, jerky truncal ataxia, scoliosis (abnormal lateral curvature of the vertebral column), vacant stare, severe dementia, intermittent hyperventilation, spastic paraparesis (increased muscle tone and weakness of the lower extremities), and vasomotor disturbances of legs. Another typical symptom of RTT patients is constant hand-wringing.

The genetic basis of RTT has been difficult to determine, as most RTT cases are sporadic. The simplest model is that RTT is an X-linked dominant trait with lethality in hemizygous males. Non-random (skewed) X-inactivation in asymptomatic mothers of RTT patients supports this model. A small number of familial cases have helped to locate the putative RTT gene to Xq28 by analysis of shared haplotypes in affected sisters and half-sisters.

Mouse methyl-CpG-binding protein 2 (Mecp2) gene is located in a $40 \mathrm{~kb}$ interval between the L1Cam and Rsvp genes on the $\mathrm{X}$ chromosome. This region is syntenic to human $\mathrm{Xq} 28$ and, with the exception of the F8A gene, locus order is conserved between two species. Mecp 2 has been shown to bind specifically to methylated DNA in vitro. The human MECP2 gene has several isoforms including a $10 \cdot 1 \mathrm{~kb}$ transcript abundant in fetal brain and a $5 \mathrm{~kb}$ transcript abundant in adult brain. The gene contains three coding exons and a giant 3'UTR; the MECP2 protein is 486 amino acids long. It contains an 85 amino acid methyl-CpG-binding domain (MBD) which binds to 5'mCpG, and a 104 amino acid transcription repression domain (TRD) which interacts with histone deacetylase and a transcription corepressor, SIN3A. This leads to deacetylation of core histone proteins and transcription silencing. Gene-targeting experiment to mutate the MECP2 gene in male mouse embryonic stem (ES) cells have shown that ES cells grow like parental lines and are capable of differentiation, but there is no fetal development. Thus, like DNA methyltransferase, $M E C P 2$ is dispensable in stem cells but essential for embryonic development.

Using heteroduplex and sequencing techniques, Amir et al (1999) have shown that mutations in the MECP2 gene cause RTT syndrome. They examined 21 sporadic and eight familial RTT cases and found 3 de novo missense mutations in $\mathrm{MBD}$, and one frame-shift and one nonsense mutation in the TRD in 5/21 sporadic cases. In one familial case (out of 8), a missense mutation was detected in halfsisters which was not present in their mother, implying that the mother was either a germ-line mosaic or had a very low level of mosaicism in her blood lymphocytes which was beyond the resolution of detection. Recent observations suggest that mutations in MECP2 account for most of the typical cases of RTT syndrome (Cheadle et al 2000). All these mutations are predicted to interfere with MECP2 function. Thus, RTT syndrome is the first human genetic disorder that is caused by mutations in a gene encoding a trans-acting factor - a factor, in other words, that has a role in the epigenetic regulation of gene expression. Cheadle et al (2000) have carried out a genotype-phenotype correlation analysis and have found that milder disease phenotypes are present in patients carrying missense mutations as compared with those with truncating mutations.

ICF (immunodeficiency, centromeric instability and facial anomalies) syndrome is a rare disorder with an autosomal recessive mode of inheritance. Patients with ICF syndrome have growth and developmental abnormalities, and facial dysmorphism such as hypertelorism (abnormal distance between two eyes), flat nasal bridge, epicanthal folds (skin folds at the corner of the eyes), micrognathia (small jaws), low set ears and tongue protrusion. Other features of ICF patients include otitis media, mental retardation, recurrent and prolonged respiratory infections, diarrhea, and infection of the skin and digestive system. Metaphase chromosome preparations from cultured peripheral blood from ICF patients show a remarkable tendency of chromosomes 1,9 and 16 to form "windmill" multiradials by interchange within decondensed heterochromatin regions. Interestingly, this feature is seen only in 
lymphocytes of ICF patients. Normal cells treated with the demethylating agent 5-azacytidine have the same effect, suggesting that demethylation of heterochromatin on chromosomes 1, 9 and 16 causes its decondensation and leads to the formation of windmill multiradials. Two years ago genetic mapping localized the ICF trait to the long arm of chromosome 20. A candidate gene, DNA methyltransferase $3 \mathrm{~b}(D N M T 3 b)$, was localized to the same area in 1999. Given the role of demethylation in the windmill phenotype in ICF patients, the DNMT3b gene became a good candidate gene for the syndrome. Soon disease-causing mutations were found in the gene in ICF patients (Xu et al 1999). These mutations do not result in a complete absence of $D N M T 3 b$ activity; this makes sense as the complete lack of this enzyme in mice is lethal in utero. Kondo et al (2000) have recently investigated the methylation abnormalities in $\mathrm{CpG}$ islands of $\mathrm{B}$ cell lines from ICF patients and their unaffected parents. They found that the methylation abnormality in ICF patients is restricted to a small portion of the genome.

With the discovery of genes for RTT and ICF syndromes, prenatal diagnosis for these syndromes in families at risk has become a reality. However, there are several questions that remain unanswered. For example, if both genes are involved in methylation-mediated transcription silencing, why do mutations in them lead to diseases with completely non-overlapping phenotypes? Could the disease phenotype of RTT patients be due to excessive non-specific 'transcriptional noise' owing to a silencing defect? And, if general transcriptional noise is the culprit, why is the brain the primary target? Demethylation leads to decondensation of heterochromatin on chromosomes 1,9 and 16, but could it cause immunodeficiency in ICF patients? What is the relationship between demethylation and craniofacial abnormalities in ICF patients? Why is the windmill phenotype seen only in blood lymphocytes? One hopes that with increasing information about the structure and function of the MECP2 and DNMT3b genes and their other family members, these questions will get answered.

\section{References}

Amir R E, Veyver I B V D, Wan M, Tran C Q, Francke U and Zoghbi H Y 1999 Rett syndrome is caused by mutations in X-linked MECP2, encoding methyl-CpG-binding protein 2; Nat. Genet. 23 185-188

Cheadle J P, Gill H, Fleming N, Maynard J, Kerr A, Leonard H, Krawczak M, Cooper D N, Lynch S, Thomas N, Hughes H, Hulten M, Ravine D, Sampson J R and Clark A 2000 Long-range sequence analysis of the MECP2 gene in Rett syndrome patients: correlation of disease severity with mutation type and location; Hum. Mol. Genet. 9 1119-1129

Kondo T, Bobek M P, Kuick R, Lamb B, Zhu B, Narayan A, Bourc'his D, Viegas-Pequignot E, Ehrlich M and Hanash S M 2000 Whole-genome methylation scan in ICF syndrome: hypomethylation of non-satellite DNA repeats D4Z4 and NBL2; Hum. Mol. Genet. 9 597-604

Xu G-L, Bestor T H, Bourc'his D, Hsieh C-L, Tommerup N, Bugge M, Hulten M, Qu X, Russo J J and ViegasPequignot E 1999 Chromosome instability and immunodeficiency syndrome caused by mutations in a DNA methyltransferase gene; Nature (London) 402 187-191

ARUN KUMAR

Department of Molecular Reproduction,

Development and Genetics,

Indian Institute of Science,

Bangalore 560 012, India

(Email, karun@hamsadvani.serc.iisc.ernet.in) 